Revista Meridiano. No. 31. Universidad de Nariño, UNED. San Juan de Pasto (ISSN: 0121-0038). (pp. 156-165). 1995.

APA citation style: Benavides B., Jorge E. (1995). Adaptación de Materiales de Lectura en Inglés: ¿Dónde empezar? Revista Meridiano. Universidad de Nariño, San Juan de Pasto. (31), 156165.

\title{
Adaptación de Materiales de Lectura en Inglés: ¿Dónde empezar? ${ }^{1}$
}

\author{
Jorge E. Benavides B. ${ }^{2}$ (joelbebu@gmail.com) \\ Departamento de Lingüística e Idiomas \\ Universidad de Nariño, Colombia
}

\section{INTRODUCCIÓN}

A menudo pensamos que los profesores de Inglés solamente deben involucrase con los diferentes aspectos de la enseñanza-aprendizaje de un idioma extranjero. Sin embargo, como investigadores potenciales en el área, el profesor de inglés además de conocer acerca de los aspectos mismos de enseñanza-aprendizaje tiene que saber cómo seleccionar y adaptar materiales de lectura, por ejemplo.

Sin embargo antes de intentar empezar a adaptar este tipo de materiales, debemos considerar algunas etapas iniciales que garantizan que el proceso sea el más adecuado. Necesitamos saber por ejemplo, quiénes y cómo son los estudiantes o aprendices, los profesores, los factores sociales involucrados, el sistema educativo, etc. En otras palabras, primero debemos analizar las necesidades del medio para el cual se van a implementar y utilizar los materiales.

Los pasos iniciales para tenerse en cuenta antes de iniciar esta etapa pueden describirse como sigue: (adaptado de 'Course Design', Dubin and Olshtain, 1986).

\footnotetext{
${ }^{1}$ Este artículo se presentó en la conferencia dictada durante el XVII Seminario Regional de Profesores de Inglés, ASOCOPI. Pasto, 6-8 de Noviembre 1991.

${ }^{2}$ Licenciado en Inglés-Francés de la Universidad de Nariño. Máster en Lingüística Aplicada para TEFL de la Universidad de Ohio, Athens, E.E.U.U. Profesor tiempo completo de la Universidad de Nariño y hora catedra de la Universidad Mariana. Director del Capítulo Académico de Informática Educativa para Aprendizajes de Idiomas (CALL) en ASOCOPI nacional.
} 
Revista Meridiano. No. 31. Universidad de Nariño, UNED. San Juan de Pasto (ISSN: 0121-0038). (pp. 156-165). 1995.

\section{FACTORES A INCLUIR EN EL ANALISIS DE NECESIDADES.}
a. La audiencia
b. El contexto de la lengua, el contexto nacional y las actitudes sociales.
c. Necesidades reales de lectura.
- Intereses
- Aptitudes
- Disponibilidad de materiales.
d. Necesidad de materiales

Estos puntos, que comprenderían la fase de reconocimiento del problema, serían la base para realizar la fase de pre-implementación, o sea lo que comprende:
a. Conversión de necesidades en valores positivos
b. Formulación de objetivos generales
c. Implementación de materiales

\subsection{LA AUDIENCIA}

Esta es una de las preguntas más relevantes que se pueden hacer los profesores puesto que trata principalmente de quien recibe el beneficio para el cual se determinan las necesidades. Este constituye el centro del proceso enseñanzaaprendizaje. La audiencia es fácil determinarla, como en el caso de los estudiantes de bachillerato, por ejemplo.

\subsection{EL CONTEXTO DE LA LENGUA}

Dentro del aspecto del contexto de la lengua, podemos empezar a trabajar en el nuestro que sería el de la lengua extranjera, el cual determina significativamente el tipo de materiales de lectura que se necesitan para propósito de enseñanza aprendizaje. 
Revista Meridiano. No. 31. Universidad de Nariño, UNED. San Juan de Pasto (ISSN: 0121-0038). (pp. 156-165). 1995.

\subsection{EL CONTEXTO NACIONAL.}

En lo que respecta al contexto nacional, necesitamos saber cuál es el papel del idioma Inglés (o el que será en nuestro país, por ejemplo); el papel del inglés dentro de un contexto de educación, el papel del inglés en el mercado laboral, y el papel de inglés en el proceso de modernización del estado (economía, educación, ciencia e investigación, industria, etc.).

El papel del inglés en nuestro sistema educativo no es tan importante como en los otros dos aspectos ya que el medio de instrucción no es el inglés precisamente a pesar de la existencia de escuelas e institutos bilingües (un número altamente reducido y que mayormente se encuentra a nivel privado): el mercado laboral y el proceso de modernización como de explotación requieren del conocimiento del inglés ya que los comerciantes, exportadores e importadores realizan sus transacciones con documentos en inglés y sus contactos los realizan directa o indirectamente con personas que hablan esta lengua.

A este respecto, las preguntas que inmediatamente se puede hacer el profesor antes de la adaptación son:

1. ¿Qué profesionales requieren conocimientos del inglés?

2. ¿Qué aspectos o habilidades de la lengua necesitan desarrollar y con qué intensidad?

Para el proceso de modernización del país, hay aspectos directamente relacionados con la información tecnológica que se introduce en inglés y la cual necesita procesarse directamente en inglés, al menos esta es la evidencia a primera vista.

En relación a este aspecto, las preguntas que se harían para tratar de adaptar materiales de lectura serían:

1. ¿Hay disponibilidad de publicaciones tecnológicas y científicas en inglés?

2. ¿Hay catálogos o instructivos en inglés que se incluyen con equipos y maquinaria modernos?

3. ¿Existen expertos extranjeros que se traen al país? 
Revista Meridiano. No. 31. Universidad de Nariño, UNED. San Juan de Pasto (ISSN: 0121-0038). (pp. 156-165). 1995.

4. ¿Hay expertos colombianos que se envían a otros países para entrenamiento y capacitación?

Cuando analizamos el problema de qué habilidad o competencia tenemos que desarrollar teniendo en cuenta el mercado laboral, inicialmente podemos decir que en nuestro país la lectura tiene que jugar un papel importante como una habilidad a desarrollar, aun antes que la de hablar (considerando que empezamos a 'leer' nuestro entorno antes de hablar). Por otro lado la misma respuesta parece ser inevitable cuando tomamos el proceso de modernización del país. El momento parece ser el más apropiado para incrementar y mejorar las habilidades y competencias de lectura entre nosotros, nuestros estudiantes y entender la importancia de los materiales auténticos en inglés, con sus implicaciones concomitantes, parece ser decisiva en el futuro cercano.

\subsection{FORMULACION DE OBJETIVOS}

Una vez que la etapa de análisis de necesidades está terminada, tenemos que considerar tres puntos importantes, los cuales van a tenerse en cuenta cuando se implementen los objetivos generales:

(a) La naturaleza de la lectura a la luz de la teoría,

(b) La naturaleza de la selección de los textos, y

(c) Las necesidades y características de los estudiantes

La formulación real de objetivos, luego, es un asunto de tomar las necesidades como base y prever o adelantarse al producto ideal que se desea, el cual al final se estimará en términos de evaluación, o sea, el último paso en la planeación, adaptación y desarrollo de los materiales de lectura.

La selección de textos puede ser un trabajo bastante exigente ya que pueden existir muchas variables para tenerse en cuenta. Una de ellas es el nivel de los estudiantes ya sean de nivel principiante, intermedio o avanzado. Otra, es la dificultad que representa la lectura del texto en términos de vocabulario y estructura, ya sea de tipo técnico, simplificado o auténtico. Una tercera variable la constituyen los tópicos o aspectos temáticos de las lecturas. Y aún otra es la extensión de los pasajes de lectura. No hay que olvidar que estas variables deben 
Revista Meridiano. No. 31. Universidad de Nariño, UNED. San Juan de Pasto (ISSN: 0121-0038). (pp. 156-165). 1995.

tenerse en cuenta cuando se llega a la fase de la organización y secuenciación de las unidades y lecciones, por ejemplo.

\section{EL PRMER PASO:}

\subsection{DETERMINAR LAS NECESIDADES Y ACTITUDES}

Después de determinar la audiencia de nuestro proyecto, es decir, los sujetos cuyas necesidades estamos interesados en conocer (los estudiantes de nivel bachillerato en la Ciudad de Pasto, con las instituciones y profesores en segundo y tercer lugar respectivamente), necesitamos conocer sus necesidades primero $y$ luego sus actitudes.

\subsubsection{NECESIDADES}

La primera pregunta a tener en cuenta es:

¿Qué clase de medios podemos utilizar para determinar las necesidades de la audiencia con respecto al aspecto de la lectura?

Podemos empezar realizando un inventario de materiales de lectura existentes en cada institución:

Libros de referencia, libros de lectura (usualmente simplificados), libros de literatura, revistas, periódicos (materiales auténticos), enciclopedias, etc.

Las preguntas que nos ayudan a recabar más información al respecto son:

1. ¿Por quién fueron desarrollados los materiales?

2. ¿Con qué propósito se desarrollaron?

3. ¿Dónde se desarrollaron?

4. ¿En realidad satisfacen los requerimientos del programa? 
Revista Meridiano. No. 31. Universidad de Nariño, UNED. San Juan de Pasto (ISSN: 0121-0038). (pp. 156-165). 1995.

\subsubsection{ACTITUDES}

Ahora bien, ¿qué clase de medios podemos utilizar para determinar las necesidades de la audiencia, es decir, de nuestros estudiantes con respecto al aspecto de la lectura?

Podemos utilizar dos diferentes criterios para hacer frente a este aspecto. El primero es el uso de encuestas con preguntas tanto directas como indirectas, con el propósito de determinar las actitudes de los sujetos hacia la lectura. El segundo es el inventario real que mediante la observación real de los materiales de lectura podemos hacer en nuestras instituciones.

\subsection{ENCUESTAS}

Por un lado, las preguntas directas que se realizan en las encuestas serán fáciles de evaluar y determinar (a pesar de que la evaluación nunca será exacta debido a la complejidad de variables y el trabajo en sí). Un ejemplo de esta clase de preguntas podría ser.

¿Qué lengua extranjera cree Ud. que es importante leer?

¿Qué importante cree Ud. que es aprender a leer en esa lengua extranjera?

a. __ muy importante

b. __ importante

c. _no muy importante

También podemos utilizar preguntas indirectas (sin necesidad de hacer el tema del inglés explicito) para aplicar el cuestionario actitudinal.

¿Qué revista extranjera le gustaría leer?

¿Qué periódico extranjero lee Ud.?

¿Qué canciones en lengua extranjera sabe Ud.?

¿Le gustaría aprender otras canciones extranjeras por medio de la lectura? (Mencione algunas de ella)

Mencione algunos cantantes extranjeros de los cuales le gustaría aprender canciones. 
Revista Meridiano. No. 31. Universidad de Nariño, UNED. San Juan de Pasto (ISSN: 0121-0038). (pp. 156-165). 1995.

Otras preguntas directas podrían ser:

¿Le gusta leer en su clase de inglés?

¿Qué clase de lecturas le gustaría leer en inglés?

\subsection{INVENTARIO}

Una de las ventajas del inventario es que las autoridades administrativas entrar a conocer, mediante el trabajo del profesor, acerca de las necesidades reales de los materiales de lectura en lengua extranjera.

Este inventario no solo se lo aplica a la institución sino que también debe aplicarse al mismo profesor.

\section{INVENTARIO DE MATERIALES DE LECTURA EXISTENTES}

Las siguientes son las preguntas dirigidas a la institución (personal administrativo de centros de recursos y bibliotecas) y a los profesores.

\subsection{PARA LA INSTITUCIÓN}

1. ¿Con cuántos textos de inglés cuenta la institución en los que hayan lecturas aparte de otros aspectos?

2. ¿Cuántos libros (no textos de enseñanza) en inglés posee la institución?

3. ¿Cuántos de ellos están destinados solo a la lectura en inglés? (Que básicamente contengan lecturas en inglés y se utilicen en el programa)

4. ¿Cuántos hay simplificados? (tipo de cuentos infantiles). Cuántos auténticos? Determine: El titulo

El autor o autores

La extensión (No. De páginas) 
Revista Meridiano. No. 31. Universidad de Nariño, UNED. San Juan de Pasto (ISSN: 0121-0038). (pp. 156-165). 1995.

La fecha de impresión

5. ¿Cuántos de literatura o ficción? (novelas, cuentos, aventuras)

Determine: El titulo

El autor o autores

La extensión (No. De páginas)

La fecha de impresión

6. Cuantos de tipo técnico o científico

Determine: El titulo

El autor o autores

La extensión (No. De páginas)

La fecha de impresión

\subsection{PARA EL PROFESOR}

1. ¿Cuántas publicaciones en ingles Ud. ha leído Ud.?

Enumere

2 ¿Cuántos libros de literatura en inglés posee Ud.?

3. ¿Cuántos ha leído?

4. ¿Qué clase de literatura técnica lee Ud. en ingles? (lingüística, psicología, computadores, etc.). Especifique el área

5 ¿Qué clase de lecturas le gustaría hacer?:

(Marque de 1 a 5 su grado de preferencia. 5 es más preferible)

Literatura

Documentales

Técnicas

Informática 
Revista Meridiano. No. 31. Universidad de Nariño, UNED. San Juan de Pasto (ISSN: 0121-0038). (pp. 156-165). 1995.

\title{
Entretenimiento
}

Po último es necesario que le profesor evalué sus propias necesidades en cuanto al conocimiento mismo de la lectura (teoría y práctica). Esto le permitirá realizar una actualización de los aspectos que conlleva la lectura.

\section{CONOCIMIENTO DEL PROFESOR ACERCA DE LA LECTURA}

Responda con una de las cuatro opciones las temáticas de abajo:

\section{Suficiente 2 Regular 3 Poco 4 Nada}

\author{
Teoría del proceso de la lectura \\ Modelos de lectura \\ Lectura en lengua extranjera \\ Técnicas de lectura \\ Análisis de texto \\ Velocidad de lectura \\ Técnica de compresión de lectura \\ Skimming (lectura cuidadosa) \\ Scanning (lectura rápida) \\ Evaluación de la comprensión de lectura
}

Si el profesor encuentra que su nivel requiere mejoría, entonces ya es tiempo de iniciar una revisión de los conceptos teóricos, lingüísticos, prácticos y metodológicos de la lectura antes de encaminarse hacia la fase de adaptación de materiales de lectura en inglés. 
Revista Meridiano. No. 31. Universidad de Nariño, UNED. San Juan de Pasto (ISSN: 0121-0038). (pp. 156-165). 1995.

\section{Conclusión}

Las necesidades de lectura en inglés como lengua extranjera pueden ser decididamente urgentes para nuestro mundo actual y estaríamos en mora de realizar algo al respecto en forma sistemática. Sin embargo, poco se lograría si antes no se dan lo pasos iniciales para encarar el problema desde nuestro contexto y desde la investigación exploratoria identificar las características de nuestras instituciones, de nuestros docentes y principalmente de nuestros estudiantes.

\section{Bibliografía}

Brown, Gillian. y George Yule (1983). Discourse Analysis. London: Cambridge. University Press.

Dubin, Freida. y Elite Olshtain. (1986). Course Design: Developint Programs and Materials for Language learning. Cambridge: Cambridge. University Press.

Nuttal, Chistine. (1985). Teaching Reading Skills in a Foreign Language. London: Heineman Educational Books. 